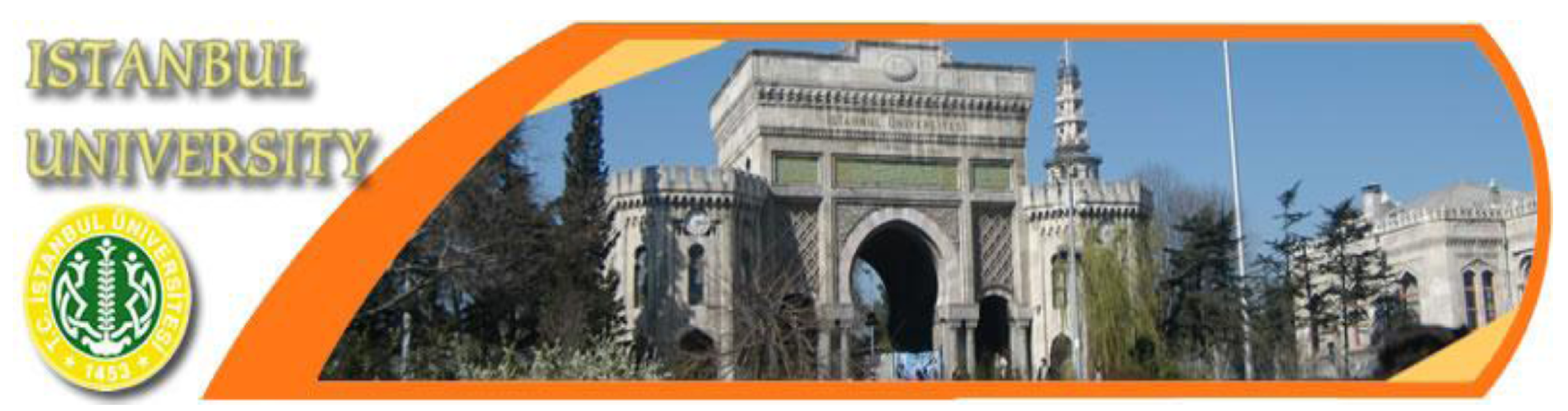

\title{
HOW CHANGED FACE OF GESTATIONAL DIABETES MELLITUS WITHIN 12 YEARS IN TURKEY
}

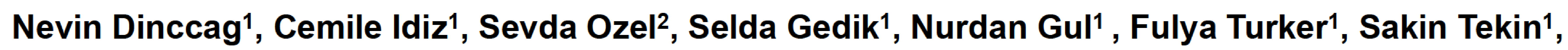 Seher Tanrıkulu ${ }^{1}$, Hulya Hacisahinoglu ${ }^{1}$, Ayse Kubat Uzum ${ }^{1}$}

1 Istanbul University, Istanbul Faculty of Medicine, Endocrinology and Metabolism Division, İstanbul/Turkey

2 Istanbul University, Istanbul Faculty of Medicine, Bioistatistics and Medical Informatics Department, Istanbul/Turkey

\section{Backgrounds and aims:}

History of Gestational Diabetes Mellitus(GDM) predisposes to carbohydrate (CHO) intolerance in the future. We aimed to determine final status of $\mathrm{CHO}$ intolerance of women with GDM who were followed between 3 months and 10 years after delivery.

\section{Materials and methods:}

240 participants with GDM history were enrolled into study and divided into two groups. [Group I: 40 women (38.0 $6.3 y$ rs) who had delivery before $11 \pm 2$ years ago, Group II: 200 women (34.0 $55.1 \mathrm{yrs}$ ) who had delivery within 3-6 months]. Final status of a participants was evaluated with $75 \mathrm{~g}$ OGTT and compared the features of groups according to possible risk factors indicating $\mathrm{CHO}$ intolerance.

Results:

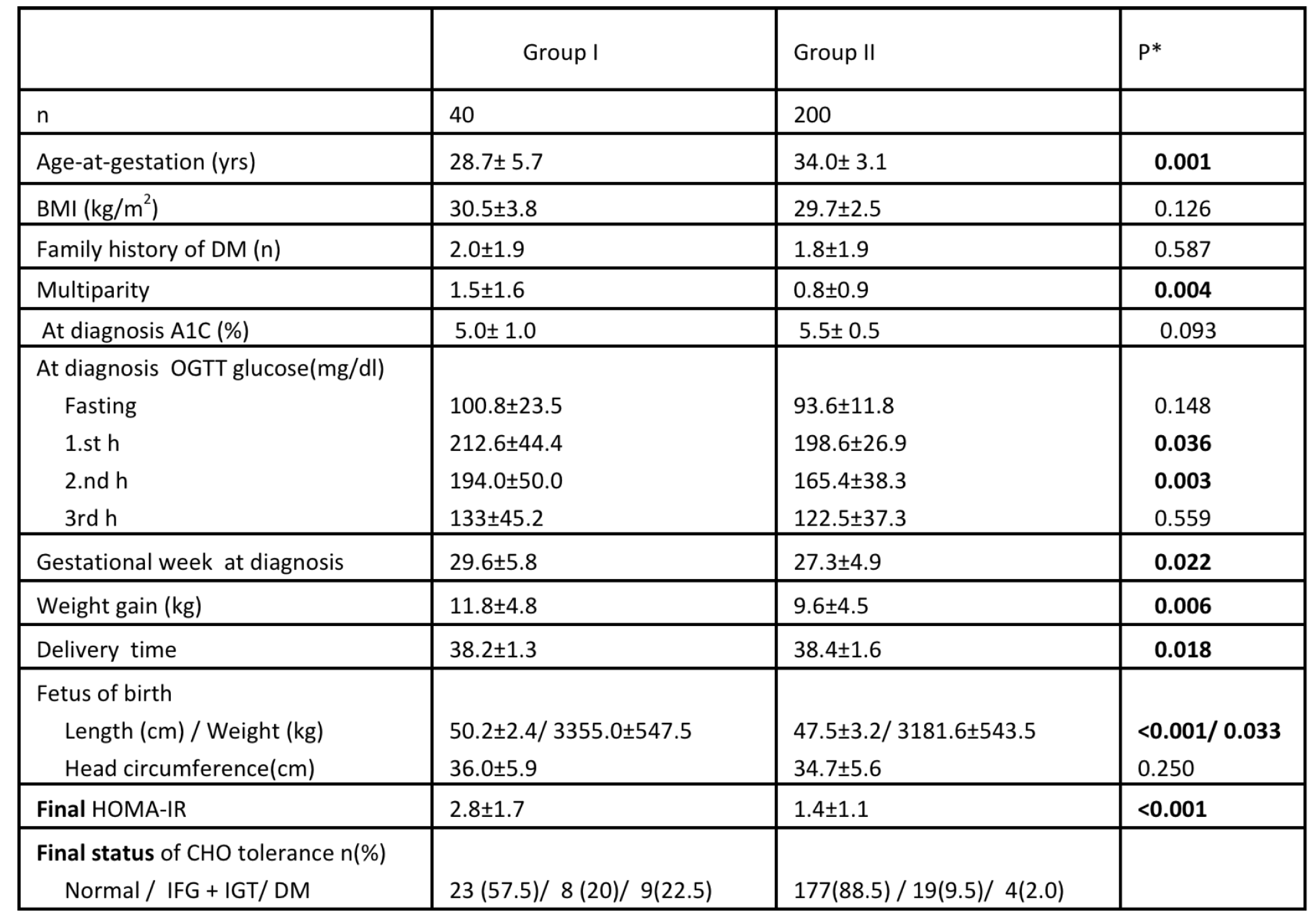

Conclusion:In our country; as compared today, twelve years ago women used to became pregnant at younger ages but diagnosis was possible at later weeks of pregnancy, delivery time was early, maternal weight gain during gestation and fetal weight at birth were high. Postpartum follow-up of GDM after delivery is important. Although CHO intolerance normalizes soon after delivery in most the women; it may sustain immediately after delivery and the frequency may be increased in the following years. Multiparity and high BMI; obtaining high glucose levels at diagnosis, are crucial predictors of development of GDM but also we can conclude that the frequency of GDM increases as follow-up years after delivery increases. 